\author{
Solange Bresson-Hadni \\ Stéphane Koch \\ Jean-Philippe Miguet \\ Michel Gillet \\ Georges-André Mantion \\ Bruno Heyd \\ Dominique-Angèle Vuitton \\ a European group of clinicians
}

\title{
Indications and results of liver transplantation for Echinococcus alveolar infection: an overview
}

S. Bresson-Hadni · D.-A. Vuitton World Health Organisation Collaborating Centre for Prevention and Treatment of Human Echinococcosis and Research Group on Alveolar Echinococcosis, University of Franche-Comté (SERF), Health and Rural Environment Research Unit,

University of Franche-Comté (SERF), Besançon, France

The authors wrote this report in collaboration with a European group of clinicians, which included the following participants. France: Jean-Pierre Crumbach, Sabine Kurz, Liver Transplant Unit, CHU Besançon; Anne Minello, Patrick Hillon, CHU Dijon and Liver Transplant Unit, CHU Besançon; Didier Samuel, Henri Bismuth, Centre Hepato-biliaire, Hopital Paul-Brousse, Paris; Maxime Audet, Philippe Wolf, Daniel Jaeck, Strasbourg; Patrick Boissel, Nancy; Filomena Conti, Yves Chapuis, Hopital Cochin, Paris; Jean Chipponi, Clermont-Ferrand; Michel Pouyet, Hôpital de la Croix Rousse, Lyons; Martine Neau-Cransac, Jean-Yves Lacut, Jacques Carles, Bordeaux; Belgium: Jean Van de Stadt, Yves Carlier, Brussels; Switzerland: Philippe Morel, Gilles Mentha, Petro Majno, Geneva; Germany: Markus Golling, Gerd Otto, Heidelberg; Juergen Klempnauer, Karl Oldhafer, Hanover; Ewert Schutte-Frohlinde, Peter Vorwald, Munich; Olaf Guckelberger, Berlin; Carl Allers, Frankfurt; Johannes Scheele, Jena; Tobias Beckurts, Cologne

S. Bresson-Hadni ( $)$

Division d'Hepato-Gastroentérologie, Département de Médecine Interne, Hopital Universitaire Cantonal de Genève, Rue Micheli du Crest, Geneva, Switzerland e-mail: solange.bresson-hadni@ufcchu.univ-fcomte.fr

S. Bresson-Hadni · S. Koch - J.-P. Miguet G.-A. Mantion - B. Heyd · D.-A. Vuitton Liver Transplant Unit, CHU Besançon, Besançon, France
M. Gillet

Liver Transplant Unit,

CHUV Lausanne,

Lausanne, Switzerland

\begin{abstract}
Background: Alveolar echinococcosis (AE) of the liver, caused by the larval stage of the fox tapeworm Echinococcus multilocularis, has the characteristics of a slowgrowing liver cancer. It is one of the rare parasitic diseases for which a parasitolytic drug is not yet available, and $\mathrm{AE}$ is lethal in the absence of appropriate therapeutic management. Complete surgical resection of the parasite at an early stage of infection provides favourable prospects for cure, but, due to a long clinical latency, many cases are diagnosed at an advanced stage, so that partial liver resection can be performed in only $35 \%$ of patients. Benzimidazole (BZM) treatment is given in inoperable cases but these compounds are only parasitostatic, and lifelong therapy is required. During the past 20 years some centres have considered liver transplantation (LT) for the treatment of
\end{abstract}

incurable AE. Methods: Our review summarizes the results of this experience based on a series of 47 European patients who received transplants between 1985 and 2002, tries to specify the real place of LT for $\mathrm{AE}$, and underlines the measures that could be undertaken in the future to improve the results. Results: Fiveyear survival was $71 \%$. Five-year survival without recurrence was $58 \%$. Major technical difficulties related either to previous laparotomies or to the loco-regional involvement were observed. The nine early deaths concerned AE patients with a long past-history of symptomatic $\mathrm{AE}$ (iterative cholangitis,

secondary biliary cirrhosis). Five late deaths were directly related to ongoing $\mathrm{AE}$, located in the brain in three cases, a very rare $\mathrm{AE}$ location that was not investigated before LT in these patients. Conclusions: In general, the pre-LT screening for distant AE metastases appeared insufficient in this series. Heavy immunosuppressive schemes, absence or delayed re-introduction of BZM after LT have clearly played a role in this unfavourable course. This unique experience indicates that, despite major technical difficulties, LT for incurable AE is feasible and could be discussed in very symptomatic cases. Before LT, interventional radiology should be preferred to repeated laparotomies. Pre-LT and post-LT BZM treatment is mandato- 
ry. A careful evaluation of possible distant metastases should be done before the decision for LT is made. After LT, the possibility of an ongoing $\mathrm{AE}$ must be permanently kept in mind. This could be reduced by lightening the immunosuppressants, carefully following the specific circulating antibodies, and applying a systematic radiological evaluation, not only to the graft but also to the lungs and the brain.
Keywords Alveolar echinococcosis $\cdot$ Echinococcus multilocularis - Treatment · Liver transplantation $\cdot$ Benzimidazoles

\section{Introduction}

Alveolar echinococcosis (AE) of the liver is a rare parasitic disease due to the intra-hepatic development of Echinococcus multilocularis larvae. It behaves like a slow-growing liver cancer [1]. Moreover, extra-hepatic extension of the parasitic disease may be possible, either by loco-regional progression, or by a haematogenous spread [1]. It might seem quite surprising for liver transplantation (LT) followed by lifelong immunosuppressive therapy to be considered for an infectious disease that sometimes affects extra-hepatic organs. However, AE is one of the sole parasitic diseases with no parasitolytic medication able to destroy the larvae and cure the patient. Moreover, until recently, due to the long clinical latency, diagnosis of advanced $\mathrm{AE}$ was the rule [2]. In these severe cases, not accessible to partial curative surgery, invasion of the bile ducts and vessels and necrosis in the central part of the parasitic lesion lead to very severe complications, such as cholangitis, liver abscesses, septic shock, portal hypertension and biliary cirrhosis, which can seriously affect not only the quality of life of these generally young patients but also their survival. In such patients, LT may offer the only chance for survival and cure, and this procedure has been proposed in advanced $\mathrm{AE}$ cases since 1985 [3, 4, 5]. Concomitantly, striking changes have occurred in the diagnosis and treatment of AE $[2,6]$, which have contributed to an improvement in the status of patients affected by this parasitic disease and made the indication for LT rarer in the recent period.

The aim of this review is to give results of this ultimate treatment for $\mathrm{AE}$, based on a series of 47 European patients, and to specify the real place of LT for this disease as well as the particularities of AE patient management before and after LT.

\section{Liver transplantation in AE: which patients?}

What was observed and done?

A recent retrospective inquiry allowed us to collect data from $45 \mathrm{AE}$ patients who had been engaged in a LT procedure from 1985 to 1999 . The detailed analysis of this series has been published recently [7]. Most of the cases come from French centres, with a total of 37 patients (two-thirds of whom received transplants in Besançon,
Table 1 European experience of LT for AE: centres involved (1985-2002)

\begin{tabular}{llc}
\hline Country & Centre & Patients $(n)$ \\
\hline France & Besançon & 23 \\
& Paris (Paul Brousse) & 3 \\
& Strasbourg & 3 \\
Nancy & 3 \\
Clermont-Ferrand & 2 \\
& Paris (Cochin) & 2 \\
Germany & Lyons (Croix-Rousse) & 1 \\
& Heidelberg & 1 \\
& Hanover & 1 \\
& Munich & 1 \\
& Berlin & 1 \\
Switzerland & Frankfurt & 1 \\
& Jena & 1 \\
Belgium & Cologne & 1 \\
Total & Lausanne & 1 \\
& Geneva & 1 \\
\end{tabular}

Eastern France, located in a highly endemic area for this disease). Interestingly, the large majority of LTs took place between 1985 and 1991 (80\% of the cases) and recent contacts with these centres indicate that there were only two additional LTs for AE between 2000 and 2002. Involved centres are listed in Table 1. Patients included 25 women and 22 men. The mean age at LT was 46.5 years (range 16-67). The mean time interval between diagnosis and LT was 5 years (range $0-23$ ).

The two main indications for LT were biliary disease, related to parasitic involvement of the hilum (Fig. 1), and tumoral disease invading both lobes of the liver, with or without chronic parasitic Budd-Chiari syndrome. Some patients had two LT indications. Thus, $57 \%$ of the cases were referred for LT because of life-threatening cholangitis and/or abscesses, $30 \%$ of the cases because of secondary biliary cirrhosis, $11 \%$ for a huge bifocal $\mathrm{AE}$ involving the hilum ("tumoral" $\mathrm{AE}$ ), and, in $7 \%$ of the cases, because of chronic Budd-Chiari syndrome with involvement of the hepatic veins ("vascular" $\mathrm{AE}$ ).

Thirty four (72\%) underwent previous upper abdominal operations: an average of 2.3 operations per patients (range 0-7) were undertaken. Major extra-abdominal surgical procedures needed to control extra-hepatic AE extension were performed in five patients. Details of all these previous operations are given in Table 2. Interventional procedures were performed before LT in 35 cases. 


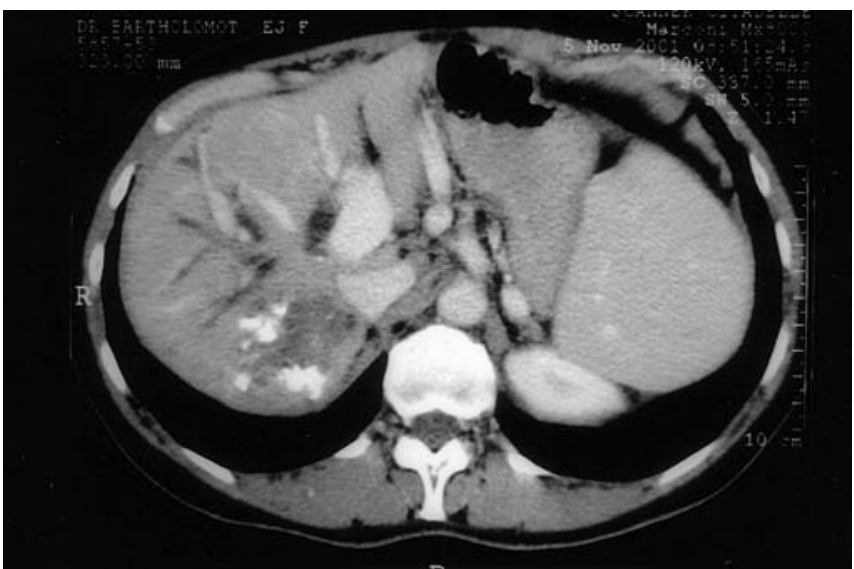

Fig. 1 A case of incurable AE in a 44-year-old woman, revealed by obstructive jaundice and complicated by acute digestive-tract bleeding, which led the treating surgeons to opt for LT. The abdominal angio-CT scan shows a typical AE lesion in the right lobe of the liver (segment VI and VII) extending to the hilum and the hepatic pedicle. There is major dilation of the intra-hepatic biliary ducts. The right portal vein is totally involved in the parasitic tissue and the portal trunk is encircled by the disease. This vascular involvement leads to portal hypertension, with huge venous collaterals and splenomegaly

Table 2 LT for alveolar echinococcosis: details of the surgical procedures needed before LT

\begin{tabular}{ll}
\hline Type of surgery & Number of patients $(\%)$ \\
\hline Abdominal operations & \\
Bilio-enteric anastomosis & $18(38)$ \\
Explorative laparotomy & $16(34)$ \\
Right or left partial hepatectomy & $10(21)$ \\
Abscess drainage & $6(13)$ \\
Atypical liver resection & $5(11)$ \\
Spleno-renal shunt & $2(4)$ \\
Extra-abdominal operations & \\
Pulmonary lobectomy & $2^{\mathrm{a}}(4)$ \\
Atrial resection & $2^{\mathrm{b}}(4)$ \\
Explorative thoracotomy & $2^{\mathrm{c}}(4)$ \\
\hline
\end{tabular}

a In two patients with AE lung metastases

${ }^{b}$ In one patient, due to recurrent parasitic clot within the right atrium responsible for iterative pulmonary embolisms

c To explore carefully the supra-hepatic inferior vena cava in two patients having parasitic extension along this vessel

In particular, there were nine radiological drainages of the bile ducts or of liver abscesses: five external drainages, two internal-external drainages and two endoscopically inserted stents.

Fifteen patients were in an extremely poor condition at the time of LT, due to a long past-history (mean duration from diagnosis to LT: 8.5 years) of symptomatic "biliary AE" with iterative cholangitis, and/or secondary biliary cirrhosis leading to ascites or digestive-tract bleeding. Nine of them died either during the operation, due to haemodynamic disorders, or in the immediate post-operative period from severe infectious complications (bacterial and/or fungal). Most of these early deaths (7/9) occurred during the first 5 years of this experience.

Among patients suffering from "vascular" or "tumoral AE", severe symptoms were observed in only half of the cases: chronic parasitic Budd-Chiari syndrome in one case leading to chronic ascites, iterative life-threatening right atrial and pulmonary embolisms in one case, huge hepatomegaly leading to abdominal pains in two other cases. Moreover, in two patients the diagnosis of $\mathrm{AE}$ was obtained by serological screening. Despite mild symptomatology, they were referred for LT because of very impressive radiological images that led to the fear of acute Budd-Chiari syndrome occurrence or extra-hepatic progression via the inferior vena cava. Unfortunately, these two patients died from chronic cholangitis (not related to recurrent AE) 3 and 5 years after they had undergone LT.

What should be done in the future?

Early deaths could certainly be avoided by the selection of patients before the terminal stage of the disease. In the case of "biliary" AE, the situation is quite similar to that of primary sclerosing cholangitis or Caroli's disease: when acute cholangitis and/or biliary cirrhosis occur, LT must be considered without delay to avoid recurrent episodes of sepsis and prolonged cholestasis that may alter nutritional status and make the peri-operative period more chaotic. When LT is being performed, such patients should certainly receive not only antibiotics but also a systematic anti-fungal peri-operative prophylaxis. In the other situations, represented by chronic parasitic Budd-Chiari syndrome and/or huge pseudo-tumoral AE, the decision to perform LT is far more difficult to make. In fact, such patients, even with very impressive radiological imagings, may be asymptomatic or pauci-symptomatic, due to the very slow progression of the parasitic larvae. The extensive use of abdominal ultrasonography and serological screening for $\mathrm{AE}$ in endemic areas has led to the more frequent discovery of such forms of $\mathrm{AE}$ in recent times $[2,8]$. The natural history of these "tumoral" and/or "vascular" AE was unknown. That explains the initial tendency for early indication for LT in such cases, due to the fear of acute Budd-Chiari syndrome occurrence or extra-hepatic progression via the inferior vena cava [9]. However, to our knowledge, acute parasitic Budd-Chiari syndrome has never been reported in AE. Moreover, a medical option, the application of continuous albendazole therapy, without the periodic interruptions of the treatment that were advised in the past and at higher dosage than previously proposed, seems to be able to stabilize such forms of incurable AE. In the Besançon centre we have chosen this policy for five pa- 
Table 3 Liver transplantation (LT) for alveolar echinococcosis: additional surgical measures during LT related to regional parasitic extension

\begin{tabular}{lc}
\hline Type of additional surgery & Number of patients $(\%)$ \\
\hline Diaphragmatic resection & $22(47)$ \\
Peri-aortic cleaning-out & $6(13)$ \\
Right adrenalectomy & $2(4)$ \\
Pericardium partial resection & $1(2)$ \\
Duodeno-pancreatectomy & $1(2)$ \\
Right nephrectomy & $1(2)$ \\
\hline
\end{tabular}

tients with such AE localizations: they remained asymptomatic, and the lesions are stable, with a mean followup of 5 years (S. Bresson-Hadni, personal communication). If one clinical episode occurs in such cases (ascites, bleeding, or septic complication), LT must, of course, be promptly considered.

\section{Liver transplantation in AE: what surgical difficulties?}

What was observed and done?

Technical difficulties are summarized in Table 3. They were numerous, related to the regional extension of the parasitic disease and the frequent past-history of previous iterative laparotomies responsible for adherences. One patient died during the hepatectomy phase and three after the liver removal. For the engrafting procedure the supra-hepatic vena cava reconstruction required an anastomosis with the right atrial ostium in three cases. In 12 cases, due to the parasitic invasion, the proper or common hepatic artery was either resected or unusable. The arterial reconstruction was done by direct implantation on the coeliac aorta in five cases and by allograft bypass to the infra renal aorta in seven cases. The biliary reconstruction required a choledocho-jejunal anastomosis in $57 \%$ of the cases. The overall mean length of the LT procedure was $8.3 \mathrm{~h}$ (range 4-20). The mean transfusion requirement was 10.6 units (range $0-88$ ). Ten patients needed early re-operation after LT for the following reasons: peri-hepatic clotting $(n=4)$, hepatic artery thrombosis $(n=2)$, hepatic artery folding $(n=1)$, diaphragmatic rupture $(n=1)$, primary non-function leading to emergency liver re-transplantation $(n=2)$.

What could be done to prevent or solve these difficulties?

It seems obvious that many patients have been penalized by a prolonged waiting time between diagnosis of incurable AE and LT. They belong to a past period, before there was access to interventional radiology. At that time the only means to get over life-threatening infectious episodes depended on iterative palliative surgical measures. This has clearly contributed to making LT very technically difficult. Selection of incurable AE patients before the terminal stage of the disease and use of percutaneous or endoscopic procedures to temporarily overcome an infectious complication are now clearly recommended, whereas multiple laparotomies must be avoided. It should clearly reduce the per-operative difficulties and make the early post-operative period less complicated. It also seems important for one to perform a very precise evaluation of the regional extension of the parasitic disease to anticipate the per-operative difficulties, the possible additional surgical measures (diaphragmatic resection, peri-aortic cleaning-out, etc.) and to specify what type of vascular anastomosis will be preferable and where to do it. Thus, depending on AE localization, liver arteriography, magnetic resonance imaging (which appeared useful for information on hepatic veins, inferior vena cava and diaphragmatic invasion), and inferior vena cava opacification, should be part of the pre-LT evaluation.

\section{Liver transplantation in AE: what medical difficulties?}

What was observed and done?

In this series, with a median follow-up duration of 6 years, the major long-term medical concern was related to the possible continuation of the parasitic disease after LT. In fact, at the time of surgery, LT was considered as palliative in 15 patients, due to inextirpable residual parasitic tissue noticed by the surgeon, or due to known $\mathrm{AE}$ metastases: seven patients had lung metastases, and one a splenic localization. Moreover, in seven patients initially considered as cured, the morphological follow-up revealed new parasitic foci, and finally, the LT was considered as palliative in $50 \%$ of the cases. The "new" AE lesions discovered during follow-up were in the spleen in one case (Fig. 2), in the brain in three cases, and in the lung in three cases. Moreover, a recurrence in the graft itself was observed in six cases: among the patients which such a location, only two were symptomatic, with diffuse re-infection leading to abscesses. In one, the diffusion occurred a few weeks after LT, clearly through a haematogenic pathway, from a splenic location of $\mathrm{AE}$ that could not be removed during the operation [10]. In the other one, multiple $\mathrm{AE}$ lesions developed 5 years post-LT, presumably from residual diaphragmatic AE foci, shortly after a liver re-transplantation for chronic cholangitis, at the time of a heavy immunosuppressive regimen. The four remaining patients were asymptomatic: the recurrent liver AE foci were limited in size and discovered by the radiological follow-up. 


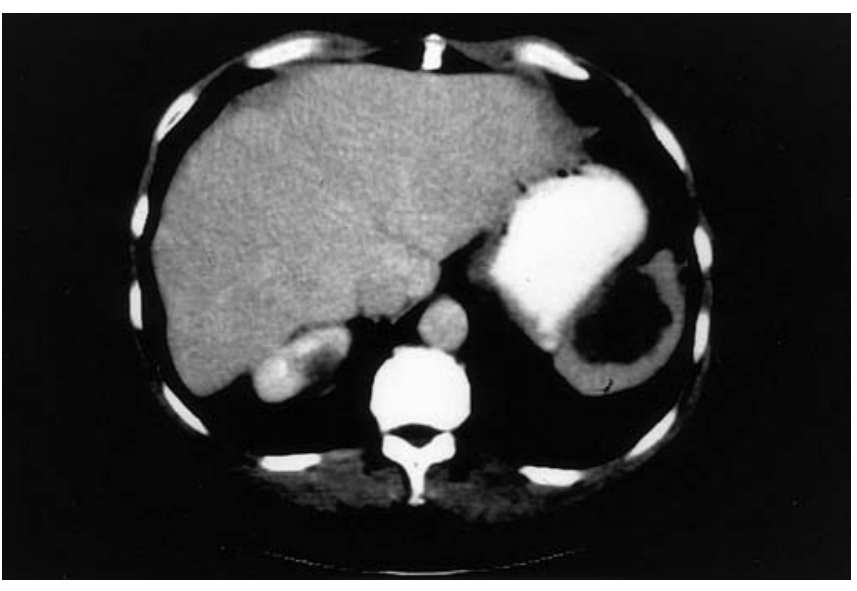

Fig. 2 Appearance of new AE localizations after LT for AE. In this patient an abdominal CT scan systematically performed 2 years after LT allowed the discovery of a splenic AE metastasis. Further introduction of $\mathrm{ABZ}$ with a current duration of 11 years led to stabilization of this lesion

Data concerning the specific serological follow-up after LT were obtained by a study performed on 15 patients in the Besançon centre [11]. An enzyme-linked immunosorbent assay (ELISA) with two parasitic antigens, a crude heterologous antigen extracted from E. granulosus $(\mathrm{EgAg})$, and the specific Em2 antigenic fraction was used for the follow-up [12]. It showed that the anti-Em2 ELISA, which is the standard test used in the patient's follow-up after partial resection for $\mathrm{AE}$ [13], did not appear useful in detecting recurrence in this particular setting. On the contrary, the EgAg ELISA, which is useless after a partial resection (most sera, even in cured patients, remained positive long after the operation) was here able to discriminate palliative and curative LT well and diagnose residual AE early. In fact, in one patient with an apparently curative LT, a secondary marked increase of anti-Eg IgG was observed at the end of the first post-operative year, 9 months before the radiological discovery of a small recurrent $\mathrm{AE}$ focus in segment VIII of the graft. On the contrary, the anti-Em2 IgG remained negative during the whole follow-up period [14].

Five late deaths were directly related to ongoing AE: three from brain $\mathrm{AE}$, at 5, 6.5, and 7.5 years; one from disseminated AE graft recurrence at 5.7 years post-LT, and one from diffuse pulmonary AE at 10 years post-LT.

Three factors have certainly played a role in explaining this high rate of continuing AE after LT: (a) the lack of precise pre-LT evaluation of the extra-hepatic extension in most cases; (b) the lack of systematic benzimidazole (BZM) use before and early after LT; (c) a tooheavy immunosuppressive scheme during the first year post-LT.

\section{Lack of precise pre-LT evaluation} of AE extra-hepatic extension

The pre-LT screening included a chest X-ray and an abdominal CT scan in all cases, but a thoracic CT scan was performed in only 20 patients (42\%) and a brain radiological investigation (CT scan or MRI) in only 12 cases $(25 \%)$.

\section{Lack of systematic BZM use before and early after LT}

Only $28(59 \%)$ of the patients received pre-LT BZM therapy, with a mean duration of 4 years. Three patients had been given flubendazole only, seven patients received mebendazole only, nine patients albendazole only and nine other patients the last two compounds, consecutively. Interestingly, one patient of the palliative subgroup who receive pre-LT mebendazole for more than 2 years had a particular evolutive pattern. Despite the per-operative observation of a small retropancreatic $\mathrm{AE}$ focus, no recurrent $\mathrm{AE}$ lesion was able to be detected at the long-term follow-up, and the patient showed rapid and persistently negative results of the specific serological tests. After LT, during the first years of this experience, due to the physicians' fear of interactions between BZM and immunosuppressants, only patients with evidence of residual AE received BZM: among the 22 patients with residual AE foci, albendazole (ABZ) was given in 19 cases; the mean time period post-LT before this drug was introduced was 28 months (range 1-115). The mean duration of therapy at the time of this study was 29 months (range 1-80). No serious adverse reactions or interactions with immunosuppressive drugs were observed. Efficacy of this treatment could be evaluated in 15 cases: the parasitostatic treatment led to a favourable course in ten patients, stabilization in seven cases and regression in three cases. In five cases, the disease progressed despite treatment.

Interestingly, among the five patients who died because of the growth of residual parasitic tissue, four did not receive pre-LT benzimidazoles. Two had adverse reactions to $\mathrm{ABZ}$ after LT (one showed digestive intolerance and after 1 month refused to take the medication; the other showed increased liver enzymes, which made the follow-up difficult and led to frequent interruption of the therapy). Among the last three patients, two exhibited progression of the residual $\mathrm{AE}$ despite long term $\mathrm{ABZ}$ therapy, and one received $\mathrm{ABZ}$ for only 18 months, when brain AE metastases were discovered, 2 years post-LT.

In the more recent period, "adjuvant" ABZ therapy, begun early after LT in apparently cured patients, was adopted in six cases. One of them is not evaluable, due to too-short a follow-up. The other five patients are alive, with a mean post-LT survival of 5 years (range 1-7) and without any evidence of recurrent AE. 
Too heavy an immunosuppressive scheme during the first year post-LT

At the beginning of this experience, there was no specific scheme of immunosuppression for these patients and the majority received a standard immunosuppressive treatment according to the period and the different liver transplant centres' protocols. Therefore, a triple immunosuppressive therapy was used in $56 \%$ of the cases, including cyclosporin or tacrolimus, azathioprine or mycophenolate and corticosteroids. The time interval before azathioprine interruption was only available in the Besançon centre. This medication was discontinued at an average of 20 months post-LT (range 2-46). Moreover, four patients from this centre were included in a multicentric trial using prophylactic anti-CD3 monoclonal antibodies for 14 days. Two other patients needed anti-lymphocyte thymoglobulins. A methylprednisolone bolus ( 1 or $2 \mathrm{~g}$ ) was given to 14 patients to treat acute rejection episodes. All six patients having had heavy prophylactic anti-rejection treatment (anti-CD3 or anti-lymphocyte globulins) had a parasitic recurrence. Recurrent AE was observed in only four of the 14 patients who received methylprednisolone bolus.

In 1992, attention was paid to the facilitating role of immunosuppressants on residual AE foci growth [14, 15], and a clear tendency to reduce the immunosuppressive regimen as early as possible was adopted by all the centres.

What could be done to prevent or solve these difficulties?

From the results of this retrospective analysis it seems very important, as for liver cancer patients, that an extensive morphological pre-LT assessment be performed, including thoracic and brain CT scans in all cases. Our experience suggests that, in the case of brain AE, LT should be ruled out. In the case of pulmonary AE metastases, the decision to maintain LT indication depends on the severity of the liver disease, the number, size and location of the metastases and the tolerance to BZM. In fact, two patients had pre-LT resection of AE lung localizations with good long-term results. In the other patients a stabilization of the lesions was obtained with long-term BZM therapy. The only patient who died from the progression of pre-existing lung metastases, 10 years after LT, did not receive ABZ because of adverse effects. Therefore, pre-LT BZM should also be recommended, firstly to assess tolerance to the drug, and secondly to try to stabilize the disease while the patient is awaiting LT. In this series, the number of patients having had pre-LT BZM is small. We cannot exclude the hypothesis that recurrence of AE after LT could have been favoured by the absence of pre-LT BZM treatment. Most of the patients of the palliative sub-group received no BZM or only a short course of it and exhibited a clear progression of the residual $\mathrm{AE}$ foci, sometimes associated with occurrence of new localizations. In contrast, the only patient of this sub-group who received MBZ for 2 years before LT has been apparently cured by LT despite the per-operative observation of a small inextirpable retro-pancreatic lesion. The hypothesis of a parasitolytic effect of MBZ on this small extra-hepatic focus, leading to a fibrous scar, can be made, as recently suggested by other teams [16, 17].

After LT, we advise the early re-introduction of BZM, before the end of the first post-operative month, when the liver tests are normalized. As recommended after a partial surgical resection [18], all patients who undergo transplantation for $\mathrm{AE}$, even if all the parasitic tissue has apparently been removed, should receive BZM for at least 2 years after LT. A regular radiological check-up (abdominal, thoracic and cerebral CT scans once a year) associated with anti-Eg antibodies follow-up, is mandatory. It seems reasonable that BZM be stopped if all these investigations prove negative at the end of the 2 nd year post-LT. Conversely, patients who show evidence of continuing AE should receive BZM indefinitely. We also recommend that the immunosuppressive regimen be kept to a minimum and that the use of anti-lymphocyte antibodies be avoided.

\section{Liver transplantation in AE: what outcome?}

What is observed, 18 years after the first attempt? Short-term and long-term survival

Kaplan-Meier actuarial survival rates were $77 \%, 71 \%$ and $49 \%$ at 1,5 and 10 years, respectively. Survival without recurrence was $58 \%$ at 5 years and $45 \%$ at 10 years. Given the severity of this disease, this result is quite satisfactory. Some patients, in a very bad condition at the time of LT, are alive and apparently cured, 20 years after the diagnosis of incurable $\mathrm{AE}$ and have returned to normal activities with a quite satisfactory quality of life. This was quite inconceivable before the LT era, and those patients have clearly benefited from this new therapeutic option. On the other hand, analysis of this series indicates that this very particular indication generates specific problems that absolutely need to be known by teams involved in the care of AE patients.

What could be done to improve the outcome; is there a future for LT in AE?

On the basis on this analysis, one must undertake a very careful management of AE patients with inoperable lesions, as soon as the diagnosis has been made, keeping 


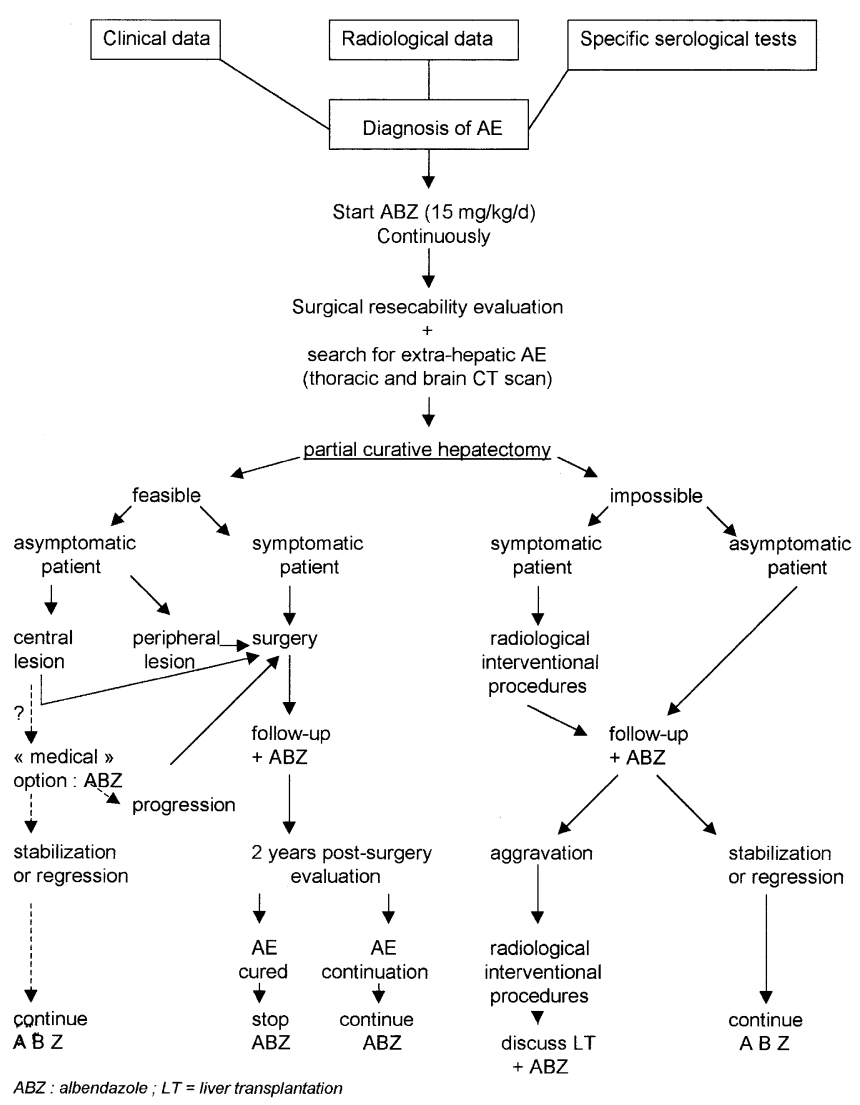

Fig. 3 Decision-maker tree for the current management of alveolar echinococcosis of the liver in mind that LT could be part of the therapeutic options taken in the future. However, the major technical difficulties during LT, the uncertainty concerning the complete cure of the disease, added to the weight of post-LT management, lead us to think that this last therapeutic option should be proposed only to patients with very symptomatic $\mathrm{AE}$. In asymptomatic or pauci-symptomatic cases, a medical option, applying ABZ therapy at higher dosage than previously proposed and using continuous and indefinite administration without the periodic interruptions that were recommended in the past, seems to be worthwhile, as it seems able to stabilize such forms of incurable AE.

In conclusion, based on this large European experience, LT for incurable AE appears feasible and should be proposed for very symptomatic patients. In the perspective of possible LT, management of septic complications should prioritize interventional radiology and avoid repeated laparotomies. Pre-LT BZM treatment is mandatory to try to stabilize the disease while the patient is awaiting LT and to check the tolerability to these compounds. Post-LT BZM, as a continuous administration for at least 2 years, is necessary after LT to minimize the risk of recurrent AE. A meticulous pre-LT evaluation of the extra-hepatic extension of the disease must be performed. Due to the high risk of AE recurrence, we recommend that the immunosuppressive regimen be kept to a minimum, anti-Eg IgG be regularly monitored and a systematic careful radiological follow-up be performed. A proposal for current $\mathrm{AE}$ management, aiming to place LT among the various options of AE patient management, is given in Fig. 3.

\section{References}

1. Bresson-Hadni S, Vuitton DA (2001) Echinococcoses. Rev Prat 51:2091-2098

2. Bresson-Hadni S, Vuitton DA, Bartholomot B, et al (2000) A twentyyear history of alveolar echinococcosis: analysis of a series of 117 patients from eastern France. Eur J Gastroenterol Hepatol 12:327-336

3. Chapuis Y, Houssin D, Brouzes S, et al (1987) Hepatic transplantation in alveolar echinococcosis. 3 attempts. Chirurgie 113:634-640

4. Mboti B, Van de Stadt J, Carlier Y, et al (1996) Long-term disease-free survival after liver transplantation for alveolar echinococcosis. Acta Chir Belg 96:229-232
5. Bresson-Hadni S, Franza A, Miguet JP, et al (1991) Orthotopic liver transplantation for incurable alveolar echinococcosis of the liver: report of 17 cases. Hepatology 13:1061-1070

6. Ammann RW, Ilitsch N, Marincek B, et al (1994) Effect of chemotherapy on the larval mass and the long-term course of alveolar echinococcosis. Hepatology 19:735-742

7. Koch S, Bresson-Hadni S, Miguet JP, et al (2003) Experience of liver transplantation for incurable alveolar echinococcosis: a 45 case European collaborative report. Transplantation 75:856-863

8. Bresson-Hadni S, Laplante JJ, Lenys D, et al (1994) Seroepidemiological screening of E. multilocularis infection in 7,884 subjects of a European endemic area of alveolar echinococcosis. Am J Trop Med Hyg $51: 837-846$
9. Gillet M, Miguet JP, Mantion G, et al (1988) Orthotopic liver transplantation in alveolar echinococcosis of the liver: analysis of a series of six patients. Transplant Proc 20:573-576

10. Slim K, Chipponi J, Pezet D, et al (1996) Récidive d'une échinococcose alvéolaire après transplantation hépatique. Lyon Chir 92:301-303

11. Bresson-Hadni S, Koch S, Beurton I, et al (1999) Primary disease recurrence after liver transplantation for alveolar echinococcosis: long-term evaluation in 15 patients. Hepatology 30:857-864

12. Gottstein B, Schantz PM, Wilson JF (1985) Serological screening of Echinococcus multilocularis infections with ELISA. Lancet i:1097-1098 
13. Gottstein B, Tschudi K, Eckert J, et al (1989) Em2 Elisa for the followup of alveolar echinococcosis after complete surgical resection of liver lesions. Trans R Soc Trop Med Hyg 83:389-393

14. Bresson-Hadni S, Miguet JP, Lenys D, et al (1992) Recurrence of alveolar echinococcosis in the liver graft after liver transplantation. Hepatology 16:279-280
15. Liance M, Bresson-Hadni S, Vuitton DA, et al (1992) Effects of cyclosporin A on the course of murine alveolar echinococcosis and on specific cellular and humoral responses against Echinococcus multilocularis. Int J Parasitol 22:23-28

16. Ammann RW, Hoffmann AF, Grimm F, et al (1998) Long-term mebendazole therapy may be parasitocidal in alveolar echinococcosis. J Hepatol 29:994-998
17. Wang X, Liu Y, Yu D (1996) Continuous therapy with albendazole for hepatic alveolar echinococcosis associated with obstructive jaundice. Clin J Intern Med 35:261-264

18. WHO Informal Working group on Echinococcosis (1996) Guidelines for treatment of cystic and alveolar echinococcosis in humans. Bull World Health Organ 74:231-242 